\title{
Patterning at 24-hour ITl: Resolution of a discrepancy more apparent than real'
}

\author{
E. J, CAPALOI AND DAVID LYNCH \\ UNIVERSITY OF TEXAS
}

Investigated was the effect of nonrewarded confinement duration on patterning in rats at a 24-hr ITI. Confinements of $120 \mathrm{sec}$. produced patterning while confinements of 30 sec. did not. These results are consistent with previous findings employing both short and long ITI.

Can rats, given an alternating schedule of rewarded (R) and nonrewarded (N) trials at 24-hr. ITI, learn to run slower on $\mathrm{N}$ than on $\mathrm{R}$ trials (pattern) without the support of external cues using only those cues provided by the $\mathrm{N}$ and $\mathrm{R}$ trials themselves? They can according to Capaldi \& Spivey (1964) who obtained patterning at 24-hr. ITI. Later Surridge \& Amsel (1965), who failed to obtain patterning at a 24-hr. ITI, came to the opposite conclusion. The results of Capaldi and Spivey, they suggested, represent only apparent patterning, produced by a failure to control differential cues on $\mathrm{N}$ and $\mathrm{R}$ trials, e.g. smell from food in goalbox, previous $S$ cues next $S, E$ cues etc. Two general comments may be offered in connection with their suggestion. Is this external cue analysis consistent with patterning investigations generally, investigations run under conditions highly similar to those of Capaldi and Spivey? Capaldi \& Spivey (1965) have shown that it is not. Nevertheless, Capaldi and Spivey did not include a control for external cues and, in retrospect, it was probably a mistake to rely entirely on past knowledge. Moreover, Surridge and Amsel failed to suggest that any of the numerous differences between the two investigators might have been responsible for the discrepancy-aside, of course, from the presumed differential cues. In this connection, Capaldi \& Spivey (1965) suggested that their failure to obtain patterning may have been related to the short (for 24-hr. ITI) nonrewarded confinement (NC) they employed (30 sec. vs. $120 \mathrm{sec}$. for Capaldi and Spivey). The abnormally long NC of $120 \mathrm{sec}$. was employed by Capaldi and Spivey because Burt \& Wike (1963) had shown patterning to be better the longer the NC. Despite such published data, Surridge and Amsel not only neglected to mention $\mathrm{NC}$ as a possible basis for the discrepancy but the value of $\mathrm{NC}$ employed was not given in their original report. ${ }^{2}$ Method

The two groups of 11 randomly assigned previously naive, 90 ( \pm 5 ) day old, male Holtzman rats received an alternating schedule of $R$ and $N$ trials at 24-hr. ITI; $R$ consisted of 30 sec. access to mash, $N$ of either 30 sec. (G30) or $120 \mathrm{sec}$. (G120) confinement in goalbox. The runway recorded times automatically (start, first 2 in., run next 52 in., and goal last 15-1/4 in.) and was modified from Capaldi and Spivey as follows. It was gray throughout. Under the entire length and width of the goalbox floor (14 $\times 4$ in.), which had $3 / 32$ in. drilled holes spaced 1 in. apart, was a $1 / 2$ in. deep metal pan filled with a semi-liquid and highly pungent mash. One goalcup ( $R$ trials) contained mash, the other (N trials) nothing. Goalcups moved noiselessly within a housing contained in goalbox; only housing visible from the alley. When the last photobeam was broken, a tightly fitting lid covering the goalcup opened automatically. Ss were grouped in squads of four each (except the last $2 \mathrm{Ss}$ ), each squad run in the same order each day. But within squads were two Ss from G30 and two Ss from G120, one S from each group getting an $R$, the other an $N$, trial on each day (first experimental trial $R$, half Ss started day 1 , half day 2). Thus there were 24 ways Ss in squads could be run, e.g. $120 \mathrm{R}, 30 \mathrm{~N}$, $30 R, 120$ N. Each order was randomly employed over 24day block. The last two Ss were run behind last squad of four, preceding and following each other an equal number of times. Ss were housed in individual cages in a separate colony room containing a masking noise from a window air conditioner. $S$ was taken from its home cage, placed in a black box for 30 sec., then placed in the runway (see Capaldi \& Spivey) and given an $\mathbf{R}$ or $\mathrm{N}$ trial. It was again placed in the black box for $30 \mathrm{sec}$. , then returned to the home cage. The goalcup was set appropriately, and then the next $S$ was taken into the experi-

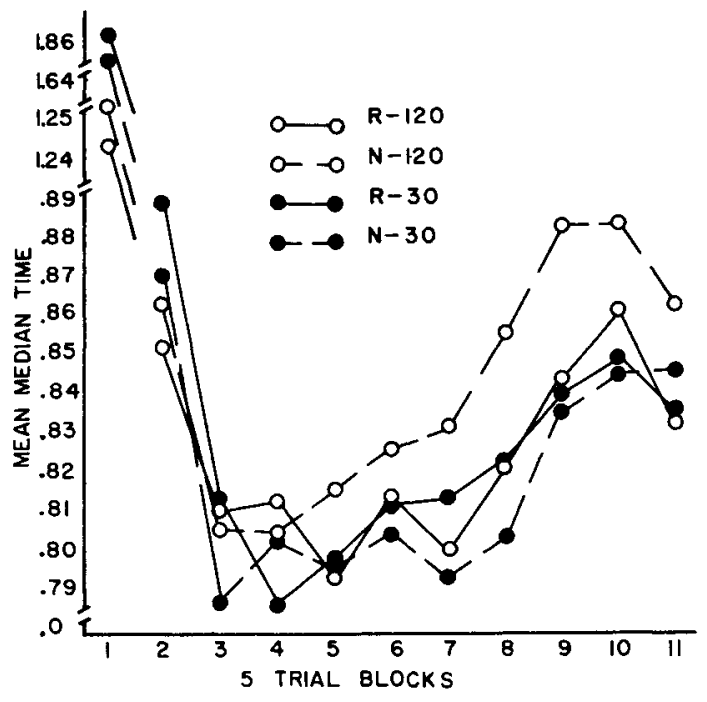

Fig. 1. Mean median time on successive blocks of $5 \mathrm{~N}$ and $5 \mathrm{R}$ trials for G30 and G120 in the run section of the alley. 
mental room and run. S was fed $10 \mathrm{gm}$ of lab-blox pellets in its home cage each day $10 \mathrm{~min}$. after being run. There were 110 trials.

\section{Results}

The median time for each $\mathrm{S}$ on each block of $5 \mathrm{R}$ and $5 \mathrm{~N}$ trials was obtained in each alley section. Figure 1 shows the mean of these medians (run-section) for G30 and G120. The results are straightforward; G30 failed to pattern whereas from about trial block 5 on, G120 patterned. On the last block of trials $R$ vs. $N$ differences were analyzed statistically. Differences for G30 were not significant in any alley section ( $F<1$ start, $<1$ run, < 1 goal). However, as in the Capaldi and Spivey investigation in G120, patterning improved from start $(F=2.50, \mathrm{NS})$, through run $(F=6.67, \mathrm{df}=1 / 10, \mathrm{p}<.05)$, to goal $(F=20.00, \mathrm{df}=1 / 10, \mathrm{p}<.01)$.

\section{Discussion}

The present results suggest that Capaldi and Spivey and Surridge and Amsel found what they ought to have found given the value of NC each employed. Since in this investigation G30 did not pattern, the patterning of G120 cannot easily be ascribed to differential smell, sounds, etc. But E may be implicated. However, E may ne implicated in practically any runway study. However, E apparently cannot be easily implicated in discretetrial operant patterning studies (e.g. Wall \& Goodrich, 1964). And Capaldi ( in press) who has advanced a theoretical analysis of $\mathrm{NC}$, has shown that operant and runway studies in many ways produce similar results. Thus the operant studies tell us more than that $\mathrm{E}$ is not necessary to produce patterning; they suggest he is not even a very important determiner of it-at least normally.

\section{References}

Burt, D. H., \& Wike, E. L. Effect of alternating partial reinforcement and alternating delay of reinforcement on a runway response. Psychol. Rep., 1963, 13, 439-442.

Capaldi, E. J. A Sequential Hypothesis of Instrumental Learning. In $\mathrm{K}$. W. Spence and J. Spence (Eds.), Recent advances in learning and motivation. New York: Academic Press, in press.

Capaldi, E. J., \& Spivey, J. E. Stimulus consequences of reinforcement and nonreinforcement: stimulus traces or memory. Psychon. Sci., 1964, 1, 403-404.

Capaldi, E. J., \& Spivey, J. E. Comment. Psychon. Sci., 1965, 3,110 and 132 .

Surridge, C. T., \& Amsel, A.. Performance under a single alternation schedule of reinforcement at 24 -hour intertrial intervals. Psychon. Sci., 1965, 3, 131-132.

Wall, A. M., \& Goodrich, K. P. Differential responding on reinforcement and nonreinforcement trials occurring in fixed repeating patterns. Psychon. Sci., 1965, 1, 193-194.

\section{Notes}

1. This research was supported in part by National Institute of Child and Health Development Research Grant HD 00949-04 to the first author.

2. We obtained the $30 \mathrm{sec}$. value from an expanded version of the Psychonomic Science Article Surridge and Amsel later submitted to J. exp. Psychol. 